Published in Stoch. Dynamics 5, 321-331 (2005).

Stochastics and Dynamics

(C) World Scientific Publishing Company

\title{
INFERENCE OF SYSTEMS WITH DELAY AND APPLICATIONS TO CARDIOVASCULAR DYNAMICS
}

\author{
A. BANDRIVSKYY, D .G. LUCHINSKY, P. V. E. McCLINTOCK \\ Department of Physics, Lancaster University, Lancaster, LA1 4YB, UK. \\ a.bandrivskyy@gmx.net,d.luchinsky@lancaster.ac.uk,p.v.e.mcclintock@lancaster.ac.uk \\ V. N. SMELYANSKIY \\ NASA Ames Research Center, MS 269-2, Moffett Field, CA 94035-1000, USA. \\ Vadim.N.Smelyanskiy@nasa.gov
}

A. STEFANOVSKA

Nonlinear Dynamics and Synergetics, Faculty of Electrical Engineering, University of Ljubljana, Tržaška 25, 1000 Ljubljana, Slovenia. aneta@osc.fe.uni-lj.si

Received (Day Month Year) Revised (Day Month Year)

\begin{abstract}
A Bayesian inference technique, able to encompass stochastic nonlinear systems, is described. It is applicable to differential equations with delay and enables values of model parameters, delay, and noise intensity to be inferred from measured time series. The procedure is demonstrated on a very simple one-dimensional model system, and then applied to inference of parameters in the Mackey-Glass model of the respiratory control system based on measurements of ventilation in a healthy subject. It is concluded that the technique offers a promising tool for investigating cardiovascular interactions.
\end{abstract}

Keywords: Stochastic delay equation; cardiovascular dynamics; Bayesian inference.

AMS Subject Classification: 34K50, 62F15, 92C30

\section{Introduction}

The inference of unobserved parameters in a stochastic dynamical model from measured time series is a problem of huge practical importance, and one that arises in a variety of different contexts. In systems with noise, unobserved parameters have to be estimated rather than evaluated, and the process of inference is therefore based on an analysis of probabilities. Thus, if some parameter is to be estimated, the whole distribution has to be computed rather than just a single number.

The well-established framework for model inference from data is based on Bayes' theorem, which relates the statistical distribution of model parameters after the measurement is made (posterior) to the statistical distribution of the model parameters prior to the measurement (prior) and to the probabilistic model of measure- 
ment itself (likelihood). The problems of how to construct the necessary probabilities, in particular the likelihood, and how to compute the posterior probability can be highly challenging. There does not yet exist any general theory of how to carry out inference for nonlinear systems with dynamical noise. Existing approaches that assume both dynamical and measurement noise rely on heavy numerical techniques, such as the Markov chain Monte Carlo (MCMC) method, to generate Bayesian conditional probabilities $[9,1]$. In the Bayesian method for estimation of noise levels suggested [4] by Heald and Stark it was assumed, however, that all other parameters of the dynamical model are known. In [2] a method for Bayesian statistical model inference was developed and applied to reconstruction of nonlinear dynamical noise-driven systems. However it relies on global optimization techniques such as simulated annealing to achieve the result. Recently a new Bayesian model inference algorithm was proposed $[10,11]$ that avoids extensive nonlinear optimization and requires only quadratic optimization in the space of model parameters to infer their mean values and variances from the trajectory measurements. A version of this algorithm for a one-dimensional noise driven dynamical system was developed in [12] in the context of the coupled-oscillator model [13] of cardiovascular dynamics.

In this paper we extend the theory [12] to encompass the problem of model identification from time-series data derived from noisy nonlinear dynamical systems with time delay, treating the delay as an unknown parameter. In Sec. 2 we outline the derivation of the inference algorithm for the particular case of a one-dimensional stochastic system with time delay, extending the results $[12,10,11]$. In Sec. 3 we verify this method numerically using a one-dimensional system with time delay. In Sec. 4 we apply it to fit the Mackey-Glass model to respiratory data, enabling us to extract important information about the cardiovascular system from measured time series. Finally, we sum up and draw conclusions in Sec.5.

\section{The inference algorithm}

We now take a one dimensional version (cf.[12]) of the inference algorithm derived $[10,11]$ and extend it to a system with time delay of the form

$$
\begin{aligned}
& \dot{x}=K(x(t), x(t-\tau) \mid c)+\xi(t), \\
& \langle\xi(t)\rangle=0, \quad\langle\xi(t) \xi(0)\rangle=D \delta(t),
\end{aligned}
$$

where $K(x(t), x(t-\tau) \mid c)$ is a deterministic drift force, $\xi(t)$ is white zero-mean Gaussian noise, $D$ is the noise intensity, and $\tau$ is the time delay. We assume that the unknown deterministic force can be represented in the form

$$
K(x(t), x(t-\tau) \mid c)=\sum_{l=1}^{L} c_{l} f_{l}(x(t), x(t-\tau)),
$$

where $f_{l}(x(t), x(t-\tau))$ are known model base functions, and $c=\left\{c_{l}\right\}$ is a set of unknown coefficients that have to be inferred from the experimentally measured time series $x(t)$. We assume that the measurement error is negligible compared to 
the intrinsic dynamical noise $\xi(t)$, and we treat the state variable $x(t)$ as directly observable.

The conditional probability density function (PDF) $L(x \mid c)$ for the system to have the trajectory $x(t)$ for a given choice of model parameters $c$ can $[9,3,8]$ be written as

$$
L(x \mid c)=\rho_{\mathrm{st}}\left(x\left(t_{0}\right) \mid c\right) \rho_{c}[x(t)] .
$$

Here $\rho_{\mathrm{st}}(x \mid c)$ is a stationary PDF of the system $(2.1)$ and $\rho_{c}[x(t)]$ is a probability density functional of the observed stochastic trajectory $x(t)$. It can be expressed through the white noise path integral using the direct interrelation between the noise variable $\xi(t)$ and $x(t)$ given by $(2.1)$.

Consider a time lattice $t_{i}=t_{0}+i h(i=0,1, \ldots, N)$, with the step $h=\left(t_{\mathrm{f}}-\right.$ $\left.t_{0}\right) / N$, and number of data points $N$. The time delay measured in $h$ and can be written as $\tau=m \cdot h$, where $m \geq 1$. Then the probability density functional for white noise defined on a time lattice $\left\{t_{i}\right\}$ can be written as

$$
\rho\left[\xi_{i}\right]=\prod_{i=m}^{N-1} \frac{1}{\sqrt{2 \pi D h}} e^{-\xi_{i}^{2} / 2 D h}=\frac{1}{(2 \pi D h)^{N / 2}} \exp \left(-\sum_{i=m}^{N-1} \frac{\xi_{i}^{2}}{2 D h}\right) .
$$

Note that the first instant of time in the equations above is shifted to $m$ to allow for the time delay. Denoting $x\left(t_{i}\right) \equiv x_{i}$ and $x\left(t_{i}-\tau\right) \equiv x_{i-m}$ we use a discrete version of the model equation (2.1)

$$
x_{i+1}-x_{i}=h K\left(\frac{x_{i+1}+x_{i}}{2}, x_{i-m} \mid c\right)+\xi_{i}
$$

to obtain the probability density functional for the stochastic variable $x$

$$
\rho_{c}\left[x_{i}\right]=\frac{1}{(2 \pi D h)^{N / 2}} J(x) \exp \left(-\sum_{i=m}^{N-1} \frac{\left[\Delta x_{i}-h K_{i, m}\right]^{2}}{2 D h}\right) .
$$

Here we denote $\Delta x_{i} \equiv x_{i+1}-x_{i}, K_{i, m} \equiv K\left(x_{i}, x_{i-m} \mid c\right)$, and

$$
J(x)=\exp \left(-\frac{h}{2} \sum_{i=m}^{N-1} \frac{\partial K_{i, m}}{\partial x}\right),
$$

is the Jacobian of transformation from $\xi$ to the $x$ variable, which implies the prepoint discretization scheme. The choice of the factor $1 / 2$ in the exponent is somewhat ambiguous due to the singular nature of white noise, and our choice corresponds to the Stratonovich prescription $[7,3]$. It is crucial to use the correct form of the Jacobian (2.3) in order to obtain a correct inference result.

The contribution to the likelihood due to the stationary probability density $\rho_{\text {st }}\left(x\left(t_{0}\right) \mid c\right)$ can be neglected if $N$ is large. Then finally the likelihood for the stochastic trajectory of the dynamical variable $x$, defined on the time lattice $t_{i}\left(t_{0} \leq t_{i} \leq t_{f}\right)$ for a certain choice of parameters $c$ may be written

$$
L(x \mid c)=\frac{1}{(2 \pi D h)^{N / 2}} \exp \left(-\frac{1}{2} \sum_{i=m}^{N-1}\left[h \frac{\partial K_{i, m}}{\partial x}+\frac{\left[\Delta x_{i}-h K_{i, m}\right]^{2}}{D h}\right]\right) .
$$


In this derivation we assumed that the time step $h$ is sufficiently small ( $h \ll$ $\left.|\partial K(x(t), x(t-\tau) \mid c) / \partial x|^{-1}\right)$ and therefore $x(t-\tau)$ was considered as a constant parameter during integration over small time step $h$. The same rule was applied to differentiation in $\partial K(x(t), x(t-\tau) \mid c) /\left.\partial x\right|^{-1}$, where $x(t-\tau)$ considered as a parameter. We note that $x(t)$ and $x(t-\tau)$ are always correlated and their independence is not required for the derivation of (2.4): the only requirement is that $\tau>h$.

Prior information about the model parameters is contained in the prior distribution $\rho(c)$. Given a set of observations, one can improve the estimate of the model parameters by computing a posterior distribution $\rho(c \mid x)$ taking advantage of Bayes' theorem

$$
\rho(c \mid x)=\frac{L(x \mid c) \rho(c)}{\int L(x \mid c) \rho(c) \mathrm{d} c} .
$$

The integral in the denominator in (2.5) ensures that the posterior probability is normalized. Clearly, equation (2.5) can be applied iteratively each time a new record of measurements is used. The prior distribution in the next iteration is just the posterior distribution from the previous iteration. For a sufficiently large number $N$ of observations in $x$, the posterior distribution $\rho(c \mid x)$ becomes sharply peaked about certain parameter values $c$ corresponding to the most probable model of the system for a given measurement set. We choose the prior distribution $\rho(c)$ as a Gaussian form with respect to the unknown parameters $c$

$$
\rho(c)=\sqrt{\frac{\operatorname{det} A_{0}}{(2 \pi)^{L}}} \exp \left[-\frac{1}{2}\left(c-c_{0}\right) A_{0}\left(c-c_{0}\right)^{T}\right] .
$$

If no initial knowledge about the model parameters is assumed, then $c_{0}$ can be chosen as a vector with arbitrary initial parameter values and $A_{0}$ can be chosen as a diagonal matrix with arbitrary but very small values of diagonal elements, creating a noninformative prior distribution.

The normalization integral in the denominator of (2.5) can readily be evaluated and the posterior probability can be written as

$$
\rho(c \mid x)=\sqrt{\frac{\operatorname{det}\left(B / D+A_{0}\right)}{(2 \pi)^{L}}} \exp (-S(c \mid x)),
$$

where the log-posterior function $S(c \mid x)$ is

$$
S(c \mid x)=G+\left(\gamma-\frac{\alpha}{D}-c_{0} A_{0}\right) c^{T}+c\left(\frac{B}{2 D}+\frac{A_{0}}{2}\right) c^{T} .
$$

Here $G$ is a constant, given by

$$
G=\left(\gamma-\frac{\alpha}{D}-c_{0} A_{0}\right)\left(\frac{2 B}{D}+2 A_{0}\right)^{-1}\left(\gamma-\frac{\alpha}{D}-c_{0} A_{0}\right)^{T}
$$


and the superscript ${ }^{T}$ denotes that a vector is transposed. We also define a scalar $R$, vectors $\alpha, \gamma$, and a matrix $B$ which are evaluated on the data set $x_{i}$ as

$$
\begin{aligned}
& \alpha_{l}=\sum_{i=m}^{N-1} \frac{1}{2}\left(x_{i+1}-x_{i}\right) f_{l}\left(x_{i}, x_{i-m}\right), \quad x_{i}=x\left(t_{i}\right), \quad l, k=\overline{1, L}, \\
& B_{l k}=h \sum_{i=m}^{N-1} \frac{1}{4} f_{l}\left(x_{i}, x_{i-m}\right) f_{k}\left(x_{i}, x_{i-m}\right), \\
& \gamma_{l}=\frac{h}{2} \sum_{i=m}^{N-1} \frac{\partial f_{l}\left(x_{i}, x_{i-m}\right)}{\partial x}, \quad R=\frac{1}{2 h} \sum_{i=0}^{N-1}\left(x_{i+1}-x_{i}\right)^{2} .
\end{aligned}
$$

The minimum of the log-posterior function (2.7) corresponds to the maximum of the posterior distribution. So, minimizing $S(c \mid x)$ with respect to the vector $c$ we obtain an estimate of the mean value of the parameters

$$
c_{1}=\left(A_{1}\right)^{-1}\left(\frac{\alpha}{D_{1}}+c_{0} A_{0}-\gamma\right)^{T}, \quad A_{1}=\frac{B}{D_{1}}+A_{0}
$$

where the matrix $A_{1}$ defines the width of the posterior distribution. For a uniform prior distribution of the noise intensity $D$ the mean value of $D$ can be estimated as

$$
D_{1}=\frac{1}{N}\left(2 R-2 \alpha c_{0}^{T}+c_{0} B c_{0}^{T}\right)
$$

In this discussion we do not consider variance of the noise intensity $D$ but note that the estimation (2.12)improves, as the inferred model parameters $c$ start to converge towards their correct values.

For a given time delay $\tau$ the algorithm (2.8)-(2.12) can be applied iteratively: the inferred mean values of the noise intensity $D_{1}$, parameters $c_{1}$ and the widths of their distributions are updated with each new block of data. Improved parameters $c_{1}$ and the matrix $A_{1}$ form the prior distribution for the next step of inference.

However, for unknown $\tau$ the log-posterior function cannot be minimized analytically in the general case when no prior information is given. It often happens, though, that the time delay may be expected to vary within some range of possible values that are known when the model is built. Thus one can define a discrete grid of the time delay parameter $\tau$, and then apply the iterative inference algorithm (2.8)-(2.12) for the set of fixed $\tau$ within a certain range. We note that this range can be arbitrary large. The only condition is $\tau>h$. For each fixed $\tau$ we find optimal coefficients $c$ and $D$. Then, using the inferred values of $c$ and $D$ we evaluate the likelihood or the corresponding log-likelihood function

$$
S_{L}(x \mid c)=\frac{N}{2} \ln (2 \pi h D)+\gamma c^{T}+\frac{R}{D}-\frac{\alpha c^{T}}{D}+\frac{c B c^{T}}{2 D} .
$$

on the data set $x_{i}, x_{i-m}$. Note that, if convergence of the parameters $c$ and $D$ has been achieved, the log-likelihood function can be simplified and evaluated as

$$
S_{L}(x \mid c)=\frac{N}{2} \ln (2 \pi h D)+\gamma c^{T}+\frac{N}{2} .
$$



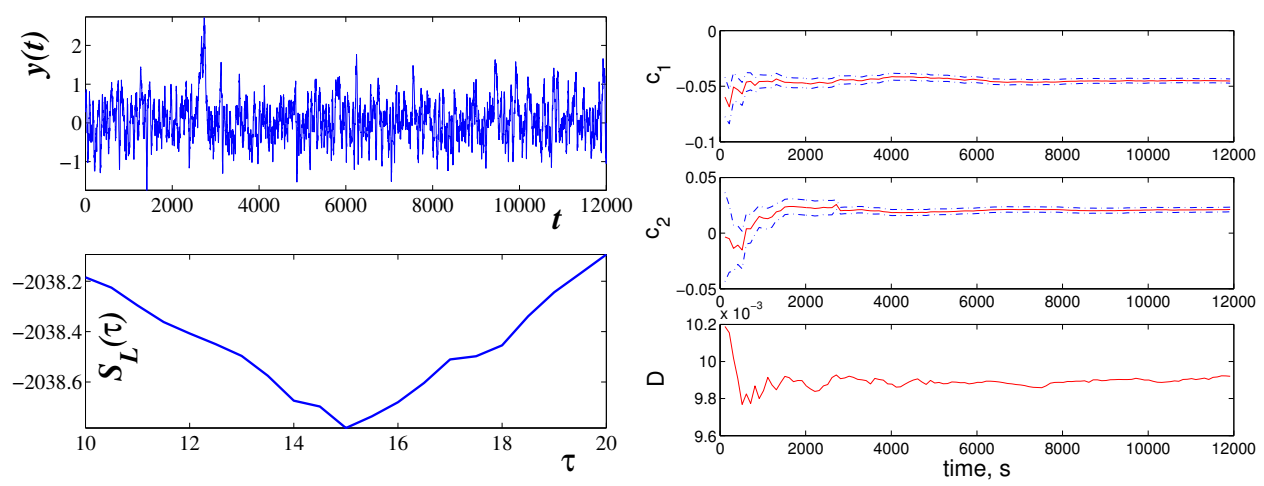

Fig. 1. Left column: the model signal $y(t)$ (top), and inference of the time delay $\tau$ (bottom). Right column: inference of all the other parameters of the model (3.13). The original (exact) model parameters were: $c_{1}=-0.05, c_{2}=0.02, \tau=15, D=0.01$.

We evaluate this function for each $\tau$ in a chosen range. Note that $S_{L}(x \mid c)$ depends on $\tau$ via dependance on other inferred coefficients and first of all via dependance on noise intensity $D$. The global minimum of the log-likelihood function reveals the optimal value of the time delay $\tau$. The corresponding to the optimal $\tau$ parameters $c$ and $D$ can be picked up as a solution to the inference problem. Thus the outlined algorithm allows for a straightforward extension to encompass inference of stochastic models with unknown time delay.

We note that one of the advantages of the suggested algorithm is its high efficiency. This makes it possible to achieve good convergence on very short time intervals (see for example parameter estimation of the Lorenz attractor in $[10,11]$. Correspondingly, we can apply the algorithm to treat slowly varying parameters, as will be explained in more detail elsewhere. Nonetheless, the general problem of analysing non-stationary data with arbitrarily strong non-stationarity in all parameters still remains an open challenge in nonlinear time-series analysis.

\section{Inference of models with a time delay}

In this section we verify numerically the algorithm outlined above. As an example we consider a simple model with a time delay

$$
\begin{aligned}
& \dot{y}=c_{1} z+c_{2} y z+\xi(t), \\
& z=y_{\tau}=y(t-\tau),
\end{aligned}
$$

where we choose $c_{1}=-0.05, c_{2}=0.02$, the noise intensity $D=0.01$, and the time delay $\tau=15$. A signal generated by the model for given parameters is shown in Fig. 1(top left). The dependance of the log-likelihood function $S_{L}(y, z \mid c)$ on $\tau$ for the model system is shown in Fig. 1(bottom left). One can see that the inferred value of $\tau$ is correct. The inference process of all the parameters of the model for 

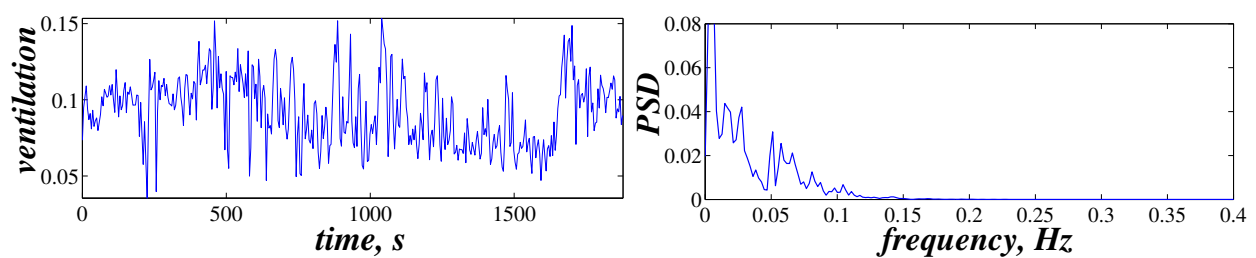

Fig. 2. The ventilation signal $V(t)$ (left) and its power spectral density (right).

$\tau=15$ is shown in Fig. 1(right). Again, one can see that the parameters converge to their correct values.

\section{Inference of the Mackey-Glass model of the respiratory control system}

Mackey and Glass proposed [6] a simple dynamical model of breathing control in which the human respiratory control was considered as a closed loop system with time delay. The model was subsequently extended [5] by Landa and Rosenblum to include the function of the brainstem respiratory center.

There are two state variables in the original Mackey-Glass model: $V$ - ventilation, which is the volume of air that passes through the lungs during a single breath multiplied by the frequency of respiration; and $x$ - the partial pressure of $\mathrm{CO}_{2}$ in the blood. The model was formulated as

$$
\begin{aligned}
& \dot{x}=\lambda-\alpha x V, \\
& V=V_{m} \frac{x_{\tau}^{n}}{\Theta^{n}-x_{\tau}^{n}},
\end{aligned}
$$

where $x_{\tau}=x(t-\tau)$, and $\tau$ is the time delay.

This simple model is able to reproduce both normal and pathological CheyneStokes breathing. The pathological or periodic breathing consists of short periods of deep and frequent breathing alternating with its complete cessation. One of the reasons for such behavior is abnormal blood circulation giving an increase in the time between oxygenation of blood in the lungs and stimulation of chemoreceptors in the brainstem. This corresponds to an increase of the time delay $\tau$ in the model (4.14)-(4.15).

In this section we show an example of inference of the slow dynamics of the respiratory control system. We slightly modify the existing model (4.14)-(4.15) and we infer its parameters from an available recording of a respiration signal. In the original and the later extended models $[6,5]$, stochastic dynamics was not considered. However noise is an inherent feature of the respiratory control system, and to take it into account we introduce a stochastic term in the equation (4.14) for $\mathrm{CO}_{2}$ concentration $x$. Further, in order to formulate a simple model for inference 

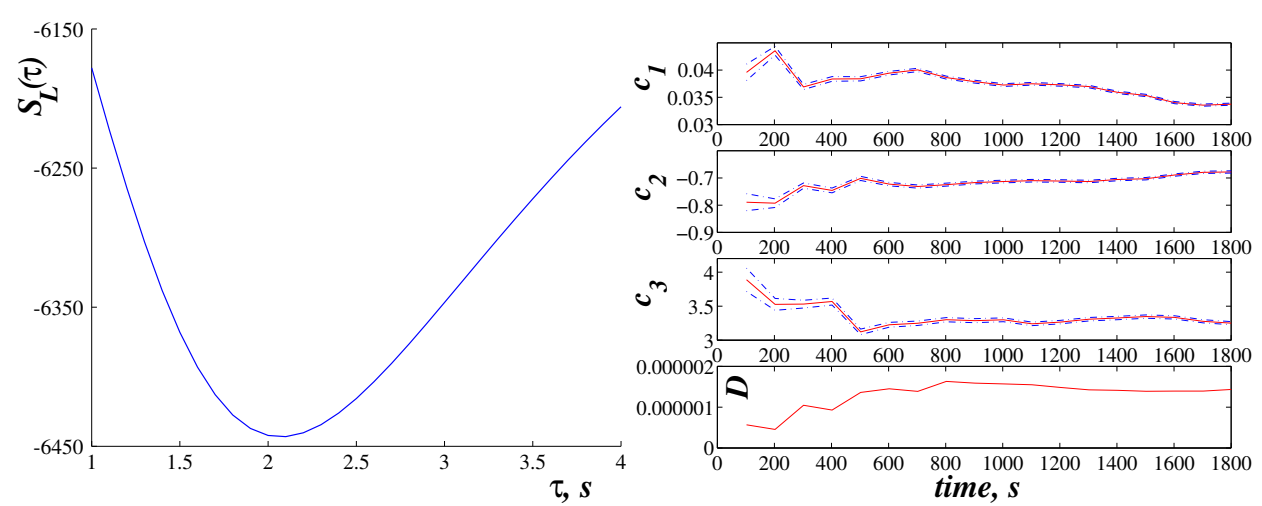

Fig. 3. Left: inference of the time delay $\tau$ for the modified Mackey-Glass model (4.18) from the constructed ventilation signal $V(t)$. Right column: inference of the other model parameters from the constructed ventilation signal $V(t)$ for the inferred time delay of $\tau=2.1 \mathrm{~s}$.

we approximate the $\mathrm{CO}_{2}$ response curve given in (4.15) as a linear function of $x_{\tau}$

$$
V\left(x_{\tau}\right)=a+b x_{\tau} .
$$

This simple approximation is justified if the control system is working close to the linear part of $V\left(x_{\tau}\right)$. The assumption is reasonable for normal and healthy breathing patterns, but it would be not correct for pathological Cheyne-Stokes breathing, when the effect of saturation in the dependence of $V\left(x_{\tau}\right)$ on $x_{\tau}$ is significant. Assuming additive white Gaussian noise $\xi(t)$, and substituting $x_{\tau}$ from (4.16) into equation (4.14) we obtain a dynamical model for ventilation

$$
\dot{V}=\lambda b+\alpha a V_{\tau}-\alpha V V_{\tau}+\xi(t)
$$

where $V_{\tau}=V(t-\tau)$ and $\tau$ is the time delay. Finally, changing variables and notation we rewrite (4.17) as

$$
\begin{gathered}
\dot{y}=c_{1}+c_{2} z+c_{3} y z+\xi(t), \\
y=V, \quad z=y_{\tau}=y(t-\tau), \quad\langle\xi(t)\rangle=0, \quad\langle\xi(t) \xi(0)\rangle=D \delta(t) .
\end{gathered}
$$

In order to apply the inference algorithm we require time series of the ventilation signal $V(t)$. We construct it from noninvasively measured respiratory effort ${ }^{\mathrm{a}} r(t)$ through the following procedure:

(i) We identify maxima and minima of the $r(t)$ signal and construct their slowly varying envelopes. We suppose that the difference $R(t)$ between these two envelope functions is proportional to the volume of the air passing through the lungs during a single breath.

(ii) We construct the continuous respiration frequency function $F(t)$, interpolating the distances between successive points. The procedure is similar to

${ }^{\mathrm{a}}$ Record TSID $=516$ from Lancaster Cardio database. 

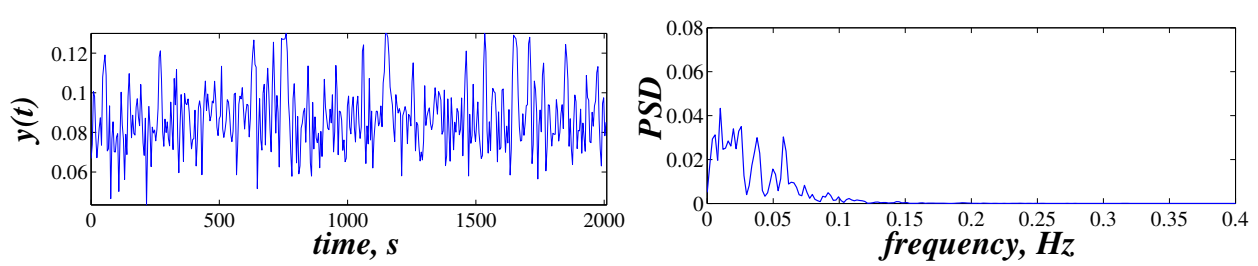

Fig. 4. Numerically generated ventilation signal $y(t)$ from the model (4.18) based on the inferred parameters (left), and its power spectral density (right).

that used for construction of the heart rate variability (HRV) signal from the ECG.

(iii) Finally, the ventilation signal $V(t)$ is computed as the product of $R(t)$ and $F(t)$. The signal and its power spectral density (PSD) are shown in Fig. 2.

Following the procedure described previously we infer parameters $c$ and $D$ for a set of $\tau$ in a range from $0 \mathrm{~s}$ to $35 \mathrm{~s}$. The behavior of the log-likelihood function near the minimum is shown in Fig. 3(left). The result of inference of the model (4.18) for the optimal (inferred) value of $\tau=2.1 \mathrm{~s}$ is shown in Fig. 3(right). The estimated parameters are: $c_{1}=0.034 ; c_{2}=-0.68 ; c_{3}=3.25 ; D=1.4 \times 10^{-6}$. In order to get some sense of whether the inference was successful, we numerically generate time series of the model (4.18) using these inferred parameters. ${ }^{\mathrm{b}}$ Time series of the model state variable $y$ and its PSD are shown in Fig. 4. This result can be compared with the original ventilation signal in Fig. 2. One can see that the model produces very similar behavior to the original ventilation signal $V(t)$, in spite of the fact that the inferred time delay parameter differs from that imposed in the original model $[6,5]$. The question, whether this is due to simplification of the model, or indeed the inferred parameter corresponds to the physiological delay in the respiratory system, we leave for future research.

\section{Summary and conclusions}

We have demonstrated a new Bayesian inference technique and shown that it can successfully be applied to stochastic nonlinear systems with delay. It enables us to extract model parameters from measured time series. We have tested the scheme on a simple one-dimensional model with delay, and applied it to the MackeyGlass model of respiratory control. For both systems the scheme converged well and yielded parameter values that were either correct or, in the latter case, plau-

\footnotetext{
${ }^{\mathrm{b}}$ Note that, because of the simplification of the model, it has unstable regions in phase space where its trajectories diverge. The stable region, where the system operates, is not well separated, and the model system can be pushed away to the unstable region by a large burst of the stochastic force $\xi(t)$. In order to eliminate this possibility in the numerical analysis we estimated the boundary of stability (which is $y_{b} \approx 0.13$ for the given signal) and imposed a limitation $y \leq y_{b}$ in the numerical algorithm.
} 
sible. It seems, therefore, that it offers a promising way of inferring physiologically relevant parameters from measured cardiovascular data.

Much remains to be done, however, before the technique can routinely be applied to the range of physiological systems that we would like to investigate. For example, the scheme needs to be extended to treat the non-white noise commonly found in physiology. In the medium/longer term, we hope to be able to infer parameters and interaction constants in the coupled-oscillator model [13] of the cardiovascular system, leading in turn to improved early diagnosis and better assessment of the efficacy treatment, based on simple noninvasive measurements.

\section{Acknowledgements}

The research was supported by the Engineering and Physical Sciences Research Council (UK), Leverhulme Trust (UK), NASA Intelligent Systems/Intelligent Data Understanding program (USA), Ministry of Education, Science and Sport (Slovenia) and INTAS.

\section{References}

1. C. L. Bremer and D. T. Kaplan, Markov chain Monte Carlo estimation of nonlinear dynamics from time series, Physica D 160 (2001) 116-126.

2. J.-M. Fullana and M. Rossi, Identification methods for nonlinear stochastic systems, Phys. Rev. E 65 (2002) 031107.

3. R. Graham, Path integral formulation of general diffusion processes, Z. Phys. B 26 (1977) 281-290.

4. J. P. M. Heald and J. Stark, Estimation of noise levels for models of chaotic dynamical systems, Phys. Rev. Lett. 84 (1999) 2366-2369.

5. P. Landa and M. Rosenblum, Modified Mackey-Glass model of respiration control, Phys. Rev. E 52 (1995) R36-R39.

6. M. C. Mackey and L. Glass, Oscillations and chaos in physiological control systems, Science 197 (1977) 287-288.

7. A. J. McKane, H. C. Luckock and A. J. Bray, Path integrals and non-Markov processes. I. General formalism, Phys. Rev. A 41 (1990) 644-656.

8. P. E. McSharry and L. A. Smith, Better nonlinear models from noisy data: Attractors with maximum likelihood, Phys. Rev. Lett. 83 (1999) 4285-4288.

9. R. Meyer and N. Christensen, Bayesian reconstruction of chaotic dynamical systems, Phys. Rev. E 62 (2000) 3535-3542.

10. V. N. Smelyanskiy and D. G. Luchinsky, Inference of stochastic nonlinear oscillators with applications to physiological problems, in Z. Gingl, J. M. Sancho, L. Schimansky-Geier and J. Kertesz (eds.), Noise in Complex Systems and Stochastic Dynamics II, (Proc. SPIE), vol. 5471 (SPIE, Bellingham) (2004) pp. 344-354.

11. V. N. Smelyanskiy, D. A. Timuchin, D. Luchinsky and A. Bandrivskyy, 
Fast Bayesian inference of stochastic nonlinear systems (available at: http://arxiv.org/abs/cond-mat/0409282), Phys. Rev. E submitted (2004).

12. V. N. Smelyanskiy, D. A. Timucin, D. G. Luchinsky, A. Stefanovska, A. Bandrivskyy and P. V. E. McClintock, Time-varying cardiovascular oscillations, in N. S. Namachchivaya and Y. K. Lin (eds.), IUTAM Symposium on Nonlinear Stochastic Dynamics (Kluwer, Amsterdam) (2003) pp. 455-464.

13. A. Stefanovska and M. Bračič, Physics of the human cardiovascular system, Contemporary Phys. 40 (1999) 31-55. 